\title{
Evaluation of different point-of-care tests to characterize the vaginal discharge of sows after parturition and parameters' correlation with subsequent reproductive performance
}

\author{
A. Grahofer (iD, T. Mäder and H. Nathues
}

\begin{abstract}
The lochia is the physiological uterine discharge post-partum, whereas abnormal fluids are often indicators of puerperal disorders in sows, which negatively influence the further reproductive performance. The aim of the study was to characterize the vaginal discharge in sows employing simple and feasible tests and to correlate the evaluated parameters with the subsequent reproductive performance of these sows. The birth process of 48 clinically healthy free farrowing sows was monitored and several parameters characterizing the vaginal discharge such as total amount, colour, amount of cells (somatic cell count) and cell characteristics (cytology) were collected daily from first to fifth day after parturition. Finally, the reproductive performance of the following gestation was evaluated and compared to the characteristics of the lochia. The amount of vaginal discharge was significantly increased on the second $(p<0.01)$, third $(p=0.019)$ and fourth $(p=0.011)$ day post-partum compared to day one. Furthermore, a decrease in the percentage of neutrophilic granulocytes from day one to three $(p=0.038)$, four $(p=$ $0.038)$ and five $(p=0.048)$ post-partum was observed. The percentage of neutrophilic granulocytes in the yellowish vaginal discharge was increased compared to whitish $(p=0.02)$ or clear $(p=0.027)$ vaginal discharge. In addition, obstetrics ( $p=0.003$ ) and an increased farrowing duration ( $p=0.017)$ significantly increased the amount of vaginal discharge. Sows with a high amount of vaginal discharge had a significant higher body temperature than sows with no ( $p=0.014)$ or low amount $(p<0.01)$ of vaginal discharge. No correlation was detected between the evaluated parameters of the lochia and the subsequent reproductive performance. It is hypothesised that the amount of vaginal discharge alone is not a predictor for the performance of sows during their next gestation. However, it might serve as indicator for acute endometritis. In summary, the different parameters of the vaginal discharge determined by means of point-of-care tests might be useful to strengthen a presumptive diagnose of endometritis in sows during the first five days after parturition.
\end{abstract}

Keywords: parturition, uterine involution, postpartal disorders, birth help, vaginal pH

\footnotetext{
* Correspondence: alexander.grahofer@vetsuisse.unibe.ch

Clinic for Swine, Department for Clinical Veterinary Medicine, Vetsuisse Faculty, University of Bern, Bremgartenstrasse 109a, CH-3012 Bern, Switzerland
}

(c) The Author(s). 2021 Open Access This article is licensed under a Creative Commons Attribution 4.0 International License, which permits use, sharing, adaptation, distribution and reproduction in any medium or format, as long as you give appropriate credit to the original author(s) and the source, provide a link to the Creative Commons licence, and indicate if changes were made. The images or other third party material in this article are included in the article's Creative Commons licence, unless indicated otherwise in a credit line to the material. If material is not included in the article's Creative Commons licence and your intended use is not permitted by statutory regulation or exceeds the permitted use, you will need to obtain permission directly from the copyright holder. To view a copy of this licence, visit http://creativecommons.org/licenses/by/4.0/ The Creative Commons Public Domain Dedication waiver (http://creativecommons.org/publicdomain/zero/1.0/) applies to the data made available in this article, unless otherwise stated in a credit line to the data. 


\section{Introduction}

Over the past three decades, the reproductive performance of sows has been considerably enhanced, due to genetic selection and improvement of housing and health management $[1,2]$. The number of weaned piglets per sow per year has risen from 20 to 30 and may increase even more in the future [1]. It is undeniable that a large litter size causes prolonged farrowing duration and decreases the health of the sows [2].

The farrowing procedure physiologically initiates an inflammatory process to enhance uterine clearance [3]. However, nowadays, prolonged parturition duration in hyperprolific sows increases the incidence of postpartal disorders, especially endometritis [4], and thereby negatively affects the subsequent reproductive cycle and performance of the sows $[5,6]$. Hence, the health status of breeding sows in the postpartal period is critical for a good reproductive performance of the herd, has a major impact on animal welfare of sows and their piglets, as well as on the economic situation of the farm [1]. It is indisputable that a diagnostic approach is required to identify pathological disorders at an early stage of any disease.

Several studies have reported that post-partum vaginal discharge occurred frequently in healthy and diseased animals [7-13], with the highest incidence between day 2 and 4 post-partum. Physiological vaginal discharge, which is watery or slightly cloudy, can be observed immediately after parturition [14-17]. An increased volume of vaginal discharge in sows is associated with endometritis, but there is no association between the occurrence of endometritis and the colour of the vaginal discharge $[14,17]$. Risk factors, such as obstetrical intervention and prolonged parturition, increase the amount of vaginal discharge during the puerperium $[10,12,18]$ and lead to a higher incidence of endometritis in sows [6].

Although characteristics of the sows' lochia after parturition, such as amount, color and $\mathrm{pH}$-value, have been described a few times in the literature, only fragmented data is available. Furthermore, most of these studies assessed the lochia in breeds with low fertility and not in modern hyperprolific sows. In particular, specific information regarding these parameters in free farrowing sows with high fertility, as shown by today's genetic lines, is not available. Therefore, the aim of this study was to characterize the lochia in sows using simple and feasible test methods during five days after parturition and to correlate these parameters with the subsequent reproductive performance of the sows.

\section{Materials and methods}

\section{Animals, husing and management}

This observational study was conducted in a farm with free farrowing pens, as part of a sow pool system. In total, 48 healthy Large White $\mathrm{x}$ Landrace sows, with a mean body condition score (BCS) of $3.4 \pm 0.3$ were included and split into three consecutive farrowing batches. The BCS of the sow was evaluated visually on an ordinal scale ranging from 1 to 5 always by the same investigator [19]. The sows were transported to the farrowing unit approximately 7 days before their estimated farrowing date (115 days of gestation) and were housed in farrowing pens, measuring $2.2 \times 2.6 \mathrm{~m}(5.72 \mathrm{~m} 2)$, part of which was a $2.75 \mathrm{~m}^{2}$ slatted floor. The room temperature in the farrowing unit was approximately $22{ }^{\circ} \mathrm{C}$. The sows were fed twice a day with a commercial feed (digestible energy $13.8 \mathrm{MJ} / \mathrm{kg}$, crude protein $22.0 \%$, crude ash $7.8 \%$, crude fat $6.0 \%$, crude fibres $5.2 \%$ ) via a liquid feeding system. The sows had free access to water, which was offered through bowl drinkers. In addition, straw was provided daily to encourage rooting behaviour (approximately $1 \mathrm{~kg} / \mathrm{sow} /$ day). The study protocol was approved by the cantonal veterinary office of Solothurn (Licence Nr. SO 18/01 29,818).

\section{Data collection}

Before parturition, the sows in each of the three farrowing batches were assigned to one of four parity groups: Group A: parity 1, group B: parity 2 and 3, group C: parity 4 and 5 , group D: parity $\geq 6$ in order to account for parity effect(s) of the evaluated parameters. Furthermore, sows that received a birth induction with PGF2 $\alpha$ (10 mg Dinoprostum intramuscular) were recorded. PGF $2 \alpha$ was intramuscularly injected on day 116 of gestation in case a sow had not farrowed until then. The data collection regarding the parturition was initiated with expulsion of the first piglet. The whole farrowing process was monitored at least every twenty minutes and data was collected. At each observation, the pen was checked for newborn piglets and placenta parts in order to retrospectively determine the duration of farrowing (first piglet to last placenta). In addition, obstetrical intervention was recorded, which was conducted if the piglet-topiglet interval was longer than $60 \mathrm{~min}$. Obstetrical intervention included manual uterine exploration and manual extraction of piglets followed by an intramuscularly injection of $20 \mathrm{IU}$ of oxytocin. With the expulsion of the last placenta, the data collection of the different parameters post-partum started. Therefore, every morning before feeding started, the vaginal discharge was examined and the quantity, colour and $\mathrm{pH}$-value was evaluated until day five post-partum. Furthermore, a cytological smear was conducted, and the number of somatic cells were evaluated. In addition, the body temperature of the sow was measured and the feed intake was assessed. Feed intake was evaluated by visual examination one hour after feeding on a dichotomous scale (normal feed intake: complete feed intake; reduced feed intake: partly 
or no feed intake). The quantity of the vaginal discharge was categorized into score 0 (no signs of vaginal discharge), score 1 (slight vaginal discharge) and score 2 (severe vaginal discharge, tail and perineal region contaminated with vaginal discharge). The colour of the vaginal discharge was classified into clear, whitish, yellowish and reddish (Fig. 1). For the evaluation of the $\mathrm{pH}$-value, two different test methods were applied. First, a pH-indicator paper (Merck Millipore, DarmstadtGermany) was used to evaluate the $\mathrm{pH}$-value of the vaginal discharge. The $\mathrm{pH}$-indicator paper was inserted into the vagina of the sow and the result was analysed and recorded immediately after removal. The second test for assessing the $\mathrm{pH}$-value was based on a cotton swab (EcoCareTM comfort, Merete Medical GmbH, BerlinGermany), which is often used in women for early detection of vaginitis, and is able to measure $\mathrm{pH}$-values ranging from 4.0 to 7.5 . The swab was inserted into the vagina of the sow and the result was analysed and recorded directly after removal.

The PortaSCC ${ }^{\circ}$ Quick test (PortaCheck, MoorestownUnited States), which has been established to determine the cell content of milk in dairy cows, was used to determine the somatic cell count of the vaginal discharge. The test was used according to manufacturer's instruction and results were recorded based on the corresponding scoring scheme. This allows measuring somatic cell estimates in a unit ' $x 1000$ cells/ml' that can also be expressed on an ordinary scale: $1(<100) ; 2$ (250); 3 (500); 4 (750); 5 (1500); 6 (> 3000).

In addition, two different test systems for the cytological examination were used in this field study. A smear of vaginal discharge was rolled across a clean microscope slide and after air drying stained with Giemsa staining. Furthermore, rapid staining technique, Testsimplets $^{\bullet}$ (Waldeck, Münster, Germany) was used. The vaginal discharge on a swab was rolled across the slides. Since the vaginal discharge was viscous, the slides had to be rinsed with distilled water before interpretation. The water led to dissolution of the coating and staining of the cells. All slides were evaluated with a light microscope in a 400-fold magnification and cells were counted in ten randomly chosen visual fields. Cells were categorized into epithelial cells and leukocytes. Epithelial cells were further differentiated into basal cells, parabasal cells, intermediate cells and superficial cells. Leukocytes were categorized into macrophages, lymphocytes, neutrophilic granulocytes, basophilic granulocytes and eosinophilic granulocytes. For the interpretation of the data, proportions between different cells were calculated. A distribution between epithelial and all nucleated cells, between leukocytes and all nucleated cells, and between neutrophilic granulocytes and all nucleated cells was considered.

The subsequent reproductive performance of sows was assessed from the farm's computerized production report. Thereby, the percentage of sows returning to oestrus within six weeks after insemination, the total born piglets and live born piglets were evaluated.

\section{Statistical analysis}

Data were collected using structured and standardized data collection forms. All data were entered into a spreadsheet program (Microsoft Office Excel 2010). Statistical processing of all data was done in NCSS 12 Data (NCSS 12 Statistical Software (2018). NCSS, LLC.

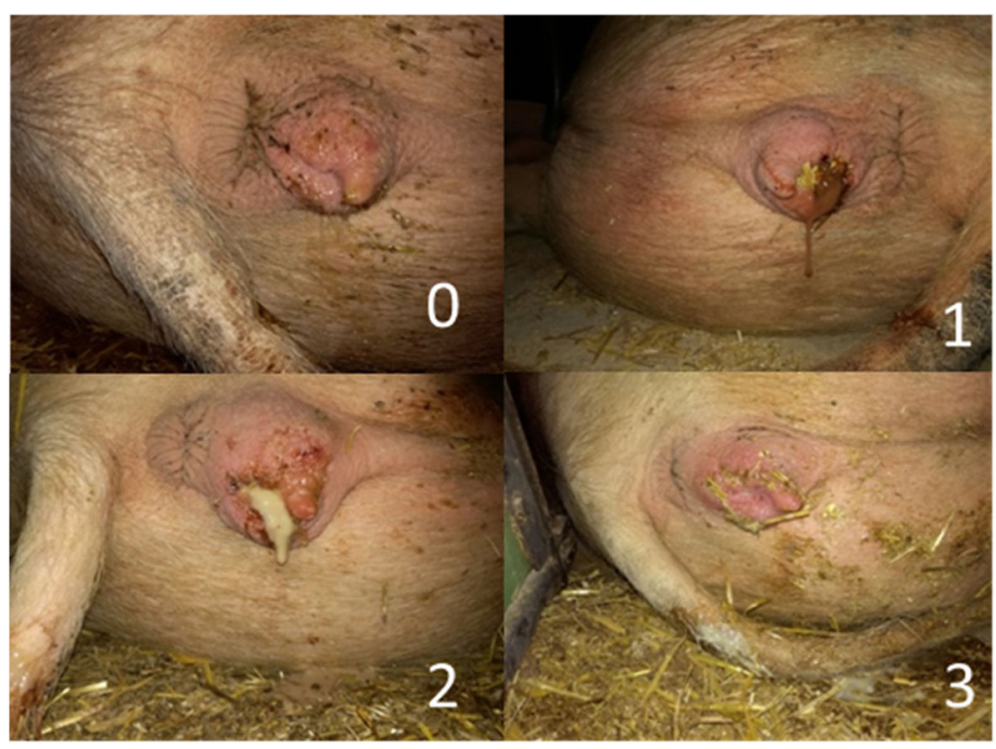

Fig. 1 Colour score of puerperal vaginal discharge. $0=$ clear, $1=$ reddish, $2=y e l l o w i s h ~ a n d ~ 3=$ whitish 
Kaysville, Utah, USA, ncss.com/software/ncss). Normality of continuous data was assessed using the ShapiroWilk test. For non-normally distributed and categorical data, the Kruskal-Wallis One-Way ANOVA was employed in more than two compared groups and the Mann-Whitney U test in two compared groups. Additionally, the Tukey Kramer Multiple-Comparison test was used for distinguishing the groups that were different from each other. For normally distributed data with equal variance the T-test was chosen. For binary data the Pearson's Chi Square Test or for $2 \times 2$ field tables the Fisher's Exact Test was employed. The correlation between the amount of vaginal discharge and the subsequent reproductive performance was analysed. Sows were assigned to either a high amount of vaginal discharge (HIGH) group or a low (LOW) group. The threshold for the HIGH group was set to the sum of all scoring values over the 5 days at $\geq 3$ because this was the mean value of all sows in this study. In all tests the level of statistical significance was considered being $\mathrm{p}<0.05$.

\section{Results}

\section{Descriptive statistics}

In total, 48 sows were included in this study. The mean parity of the study population was $3.6 \pm 2.5$ (mean \pm standard deviation) and ranged from 1 to 11 . The mean number of total born piglets was $14.8 \pm 3.0$, whereof $4.1 \%$ were stillborn. Overall, 81 samples of vaginal discharge were evaluated from 39 out of 48 sows in the early post-partum period (day 1 to 5 ). In the other nine sows vaginal discharge could not be observed at any time point. The prevalence of post-partum vaginal discharge was significantly lower on the first day postpartum (4/48 sows; $8.3 \%$ ) compared to the second (23/
48 sows; $48.0 \%$; $\mathrm{p}=0.0005)$, third $(21 / 48$ sows; $43.8 \%$; $\mathrm{p}=$ $0.019)$, and fourth $(22 / 48$ sows; $45.8 \%$; $\mathrm{p}=0.011)$ day. Further details of the prevalence of vaginal discharge are presented in Fig. 2. The evaluation of the colour of the vaginal discharge revealed that whitish vaginal discharge was most often detected with a prevalence of $40.7 \%$ ( $n=$ 33), followed by yellowish $35.8 \%(\mathrm{n}=29)$, reddish and clear $13.6 \%(n=11), 9.9 \%(n=8)$, respectively. Further details of the distribution of the colour of vaginal discharge on the different sampling days are presented in Fig. 3. No significant difference between the colour and the amount of vaginal discharge was detected.

The PortaSCC ${ }^{\circ}$ test was conducted in all sows demonstrating vaginal discharge and detailed results are displayed in Fig. 4. The somatic cell count did not significantly differ between the days of observation. Furthermore, no correlations between the somatic cell count and the amount or the colour of vaginal discharge or the vaginal $\mathrm{pH}$-value were detected.

Due to a lack of sufficient amount of material to perform both smears, only four samples were collected on the first day, 17 on the second day, 15 on the third and fourth day and eight on the fifth day post-partum. An overview of the results of the cytological examination conducted with two test systems on the different sampling days are presented in Figs. 5, 6 and 7. There was no significant difference between the two test methods in evaluating the amount of leukocytes, neutrophilic granulocytes and epithelial cell. Overall, a significant difference between the colours of the vaginal discharge and the mean percentage of neutrophilic granulocytes of both tests was detected. In sows with a clear vaginal discharge less neutrophilic granulocytes $(20.7 \% \pm 5.3)$ could be detected in the cytology compared to sows with

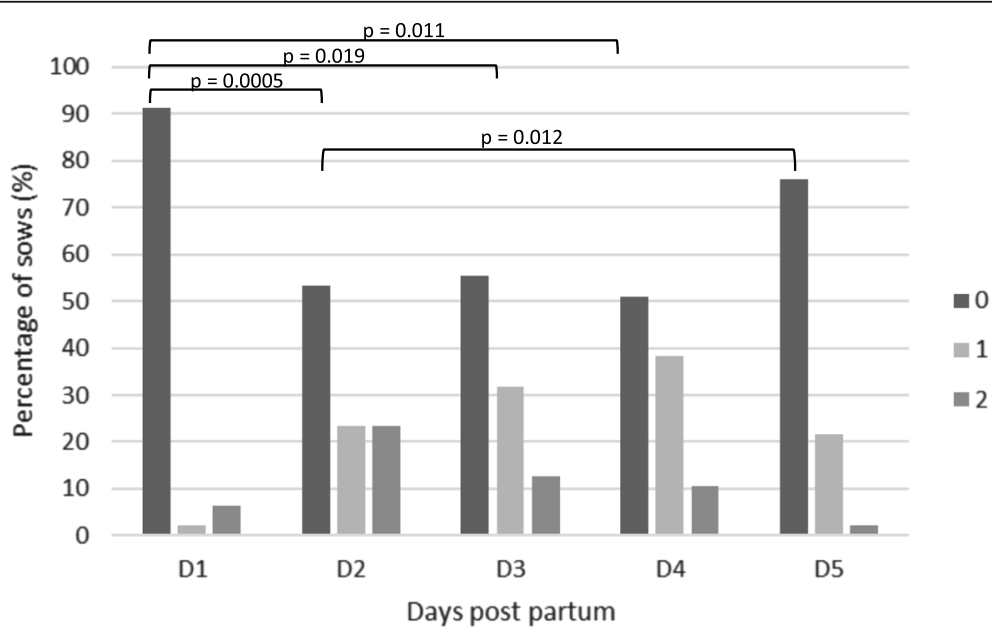

Fig. 2 Distribution of the percentage of sows according to the quantity of vaginal discharge $(0=$ no signs of vaginal discharge, score $1=$ slight vaginal discharge and score $2=$ severe vaginal discharge, tail and perineal region contaminated with vaginal discharge) from day 1 to day 5 after parturition 


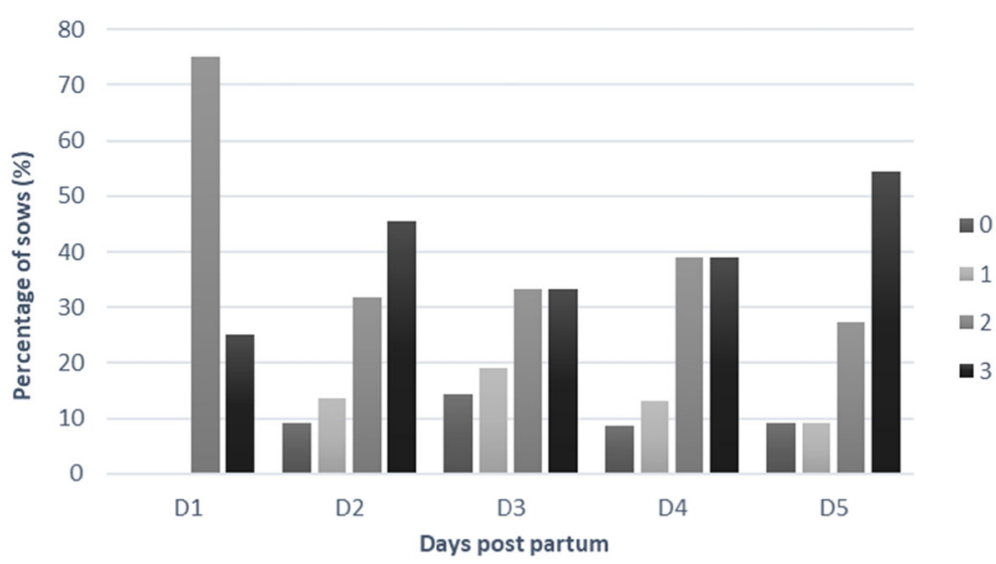

Fig. 3 Distribution of the percentage of sows with vaginal discharge according to colour of vaginal discharge $(0=$ clear, $1=$ reddish, $2=y e l l o w i s h ~$ and $3=$ whitish) from day 1 to day 5 after parturition

a reddish $(53.1 \% \pm 38.3 ; \mathrm{p}=0.019)$ and yellowish vaginal discharge $(55.9 \% \pm 24.2 ; \mathrm{p}<0.01)$. In addition, sows with a yellowish vaginal discharge had significantly more neutrophilic granulocytes $(55.9 \% \pm 24.2 ; \mathrm{p}=0.014)$ in the smear compared to sows with a whitish vaginal discharge $(37.9 \% \pm 24.0)$.

\section{Analysis of vaginal discharge traits on sow traits and birth performance}

In this study, birth induction was conducted in 16 out of 48 sows. The median farrowing duration was $211 \mathrm{~min}$ (Min: 55; Max: 525), without a significant difference between the parity groups. In 19 out of 48 sows (39.6\%), obstetrical intervention was conducted. Obstetrical intervention significantly $(\mathrm{p}=0.003)$ increased the sum of scoring the vaginal discharge in sows within the first five days post-partum (Fig. 8).

Retrospectively, 28 sows (58.3\%) were assigned to the low amount of vaginal discharge (LOW) group (sum of the scoring over five days $\leq 2$ ) and 20 sows (48.6\%) were assigned to the high amount of vaginal discharge (HIGH) group (sum of the scoring over five days $\geq 3$ ). Sows in the HIGH group showed a significantly longer farrowing duration (Median: 252; Min: 104; Max: 525) when compared to sows in the LOW group (Median: 188; Min: 55; Max: 324; $\mathrm{p}=0.017$; Fig. 9).

Further details of the study population considering parity groups, birth duration and vaginal discharge are presented in Table 1. No significant difference was detected between the LOW and HIGH group of vaginal discharge and the parity groups. Moreover, no significant differences were observed between parity, BCS, birth induction and the LOW and HIGH group of vaginal discharge.

Analysis of vaginal discharge traits on body temperature, feed intake post-partum and $\mathrm{pH}$-value of the vagina An overview of the body temperature and the feed intake during the five sampling days grouped by the amount of

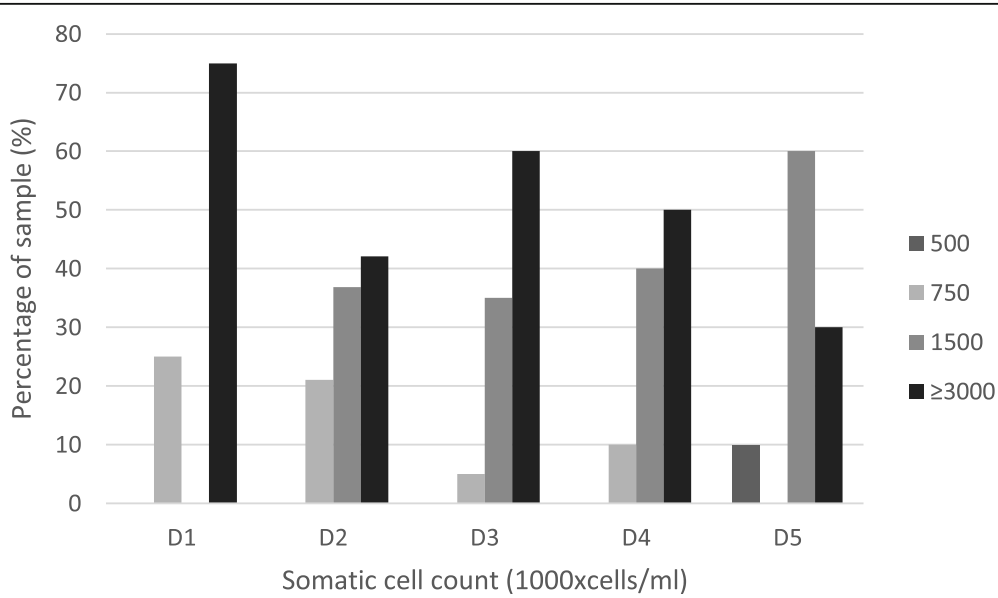

Fig. 4 Distribution of the percentage of samples collected from sows with vaginal discharge according to the somatic cell count of the lochia tested with PortaSCC $^{\oplus}$ test from day 1 to day 5 after parturition 


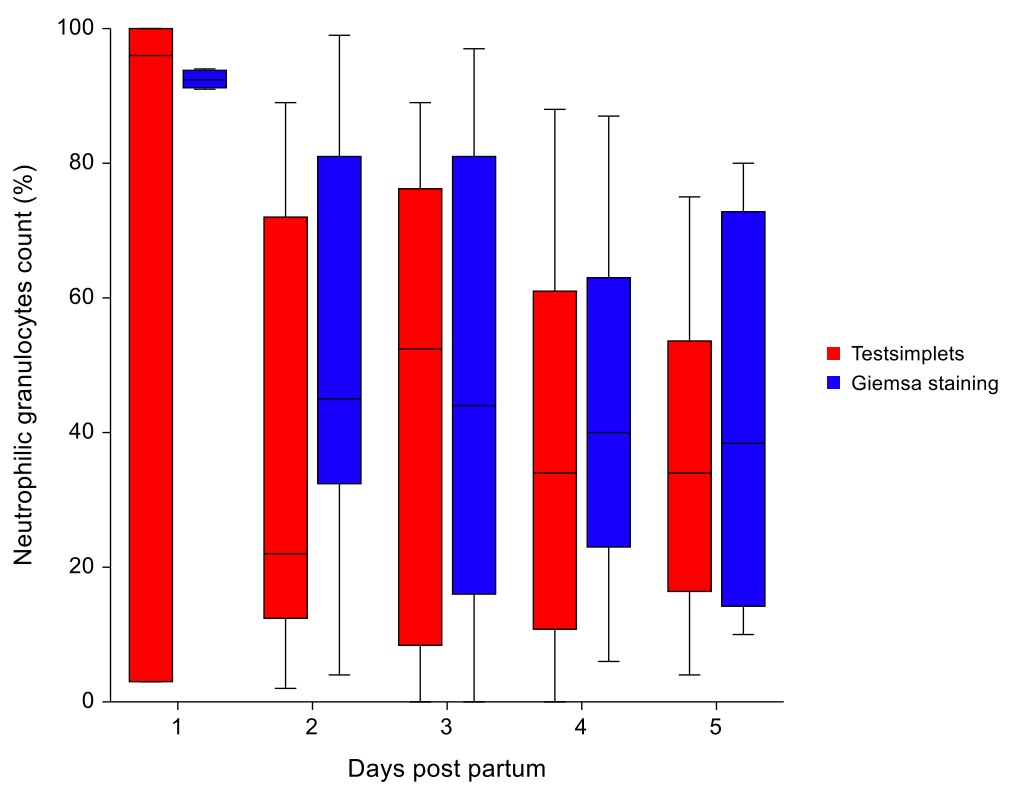

Fig. 5 Distribution of neutrophilic granulocytes in the vaginal discharge of sows evaluated with two different methods (Testsimplets and Giemsa staing) from day 1 to day 5 after parturition. The leukocytes are expressed as a percentage of the total number of nucleated cells counted

vaginal discharge is presented in Table 2. No correlation between feed intake and amount of vaginal discharge on a particular sampling day was detected. A significant difference of the body temperature in sows between day 1 after parturition (Median: $38.8^{\circ} \mathrm{C}$; Min: $37.0^{\circ} \mathrm{C}$; Max: $40.7^{\circ} \mathrm{C}$ ) and day 3 (Median: $38.3^{\circ} \mathrm{C}$; Min: $37.5^{\circ} \mathrm{C}$; Max: $39.9^{\circ} \mathrm{C}$; $\mathrm{p}=0.002$ ) and day 4 (Median: $38.3^{\circ} \mathrm{C}$; Min: $37.0^{\circ} \mathrm{C}$; Max: $\left.39.7^{\circ} \mathrm{C} ; \mathrm{p}=0.0018\right)$ was seen. In addition, sows with a severe amount of vaginal discharge had a significant higher body temperature (Median: $38.7^{\circ} \mathrm{C}$; Min: $37.8{ }^{\circ} \mathrm{C}$; Max: $40.9^{\circ} \mathrm{C}$ ) than sows with no (Median: $38.5^{\circ} \mathrm{C}$; Min: $37.0^{\circ} \mathrm{C}$; Max: $\left.41.1^{\circ} \mathrm{C} ; \mathrm{p}=0.014\right)$ or slight amount of vaginal discharge (Median: $38.3^{\circ} \mathrm{C}$; Min: $36.5^{\circ} \mathrm{C}$; Max: $39.9^{\circ} \mathrm{C}$; $\mathrm{p}<$ 0.01 ), when considering all days of observation. Details are

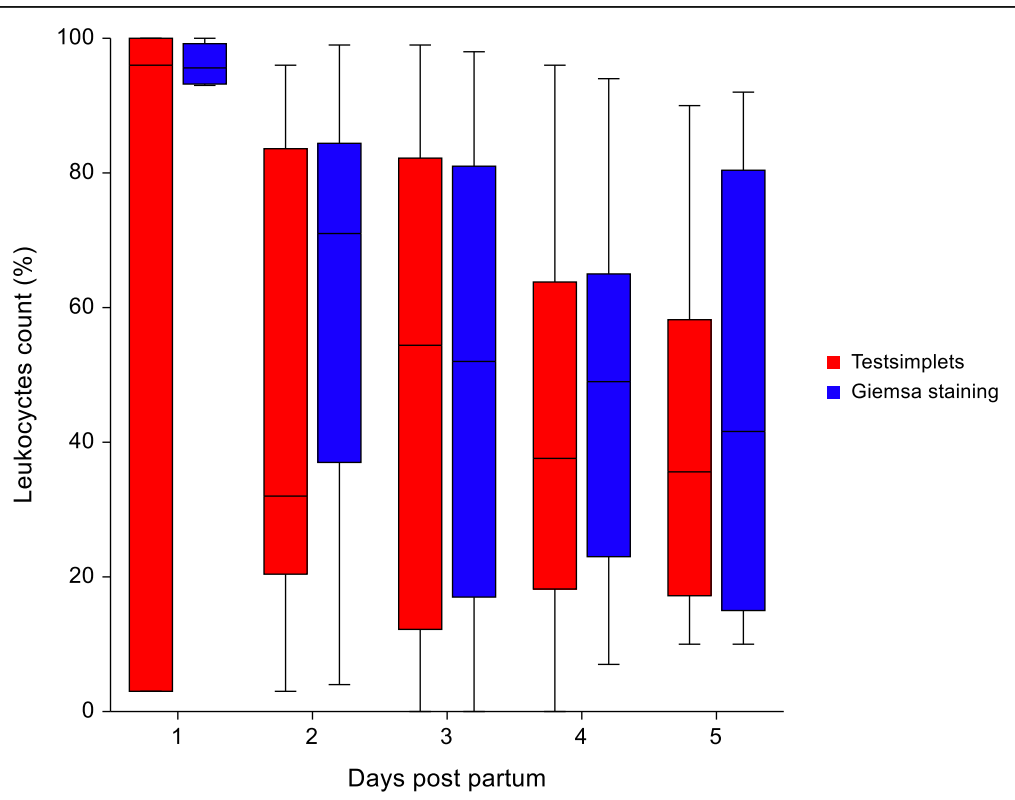

Fig. 6 Distribution of leukocytes in the vaginal discharge of sows evaluated with two different methods (Testsimplets and Giemsa staing) from day 1 to day 5 after parturition. The leukocytes are expressed as a percentage of the total number of nucleated cells counted 


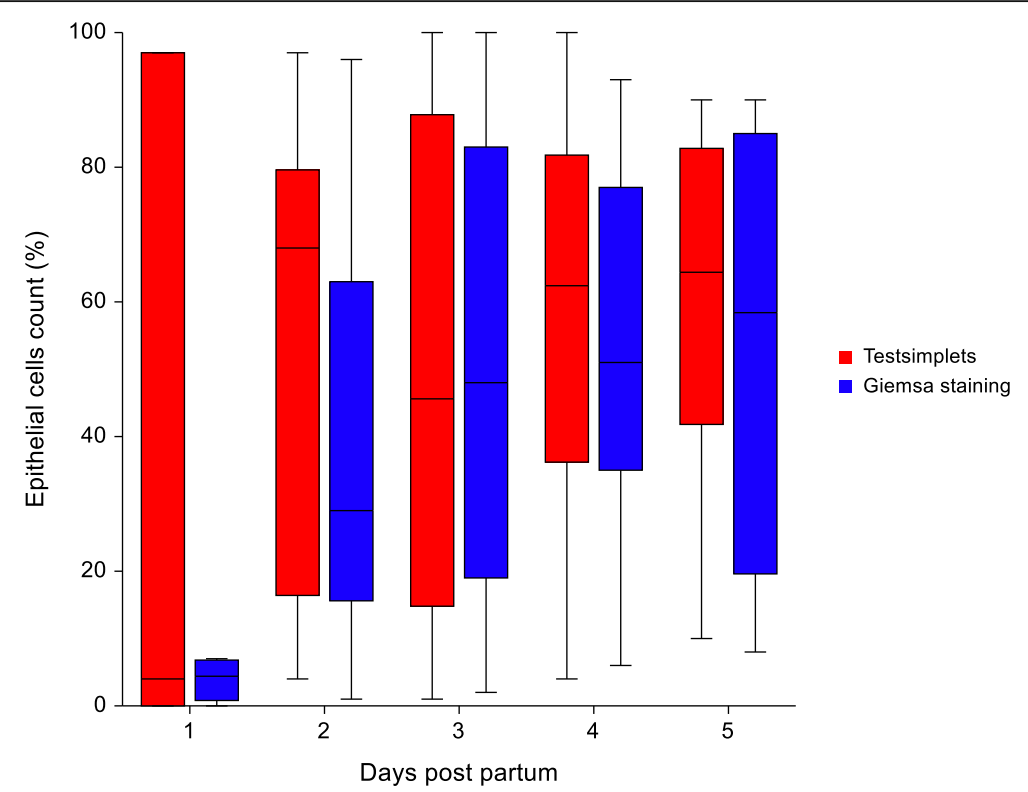

Fig. 7 Distribution of epithelial cells in the vaginal discharge of sows evaluated with two different methods (Testsimplets and Giemsa staing) from day 1 to day 5 after parturition. The leukocytes are expressed as a percentage of the total number of nucleated cells counted

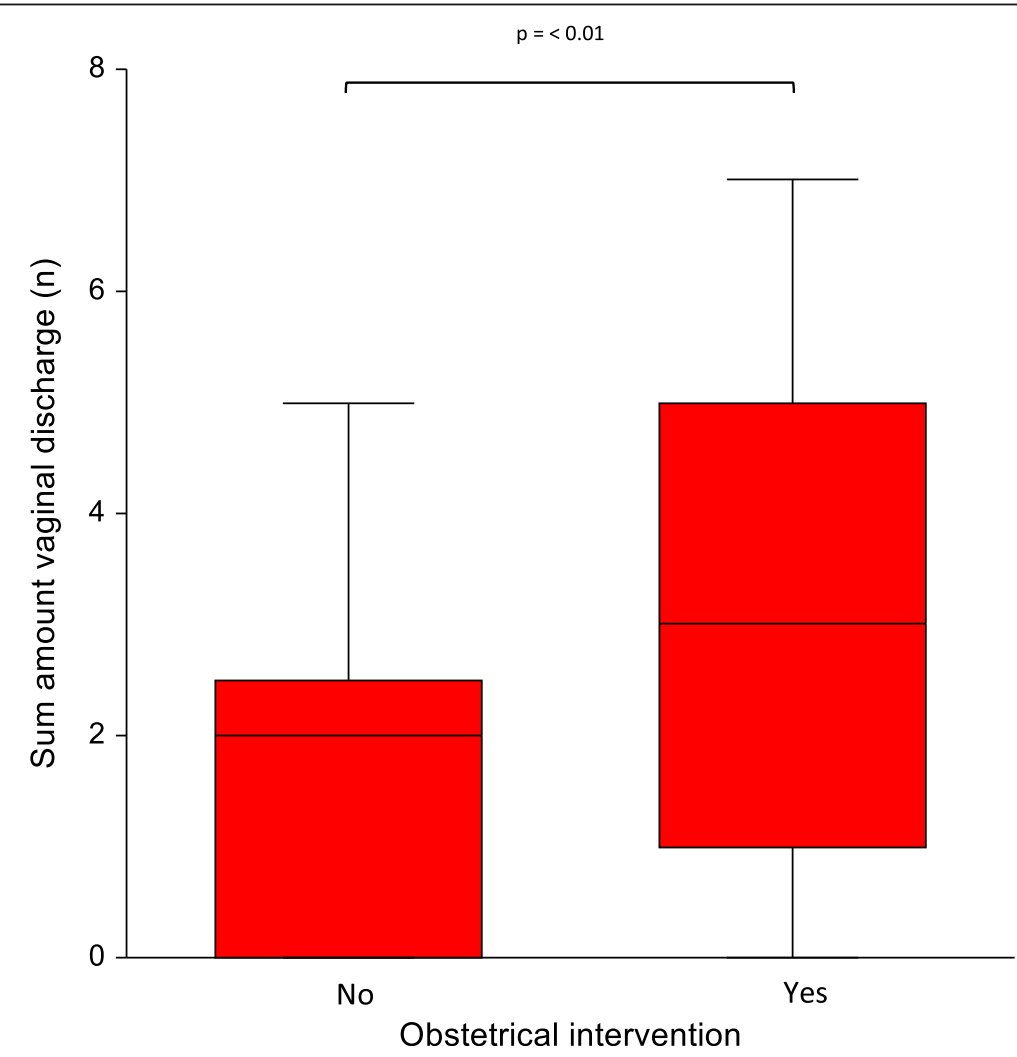

Fig. 8 Comparison of obstetrical intervention during parturition ( $\mathrm{No}=$ no measures conducted, Yes= obstetrics conducted) with the amount of vaginal discharge. The amount of vaginal discharge was calculated by summing the scores over the 5 days post-partum 


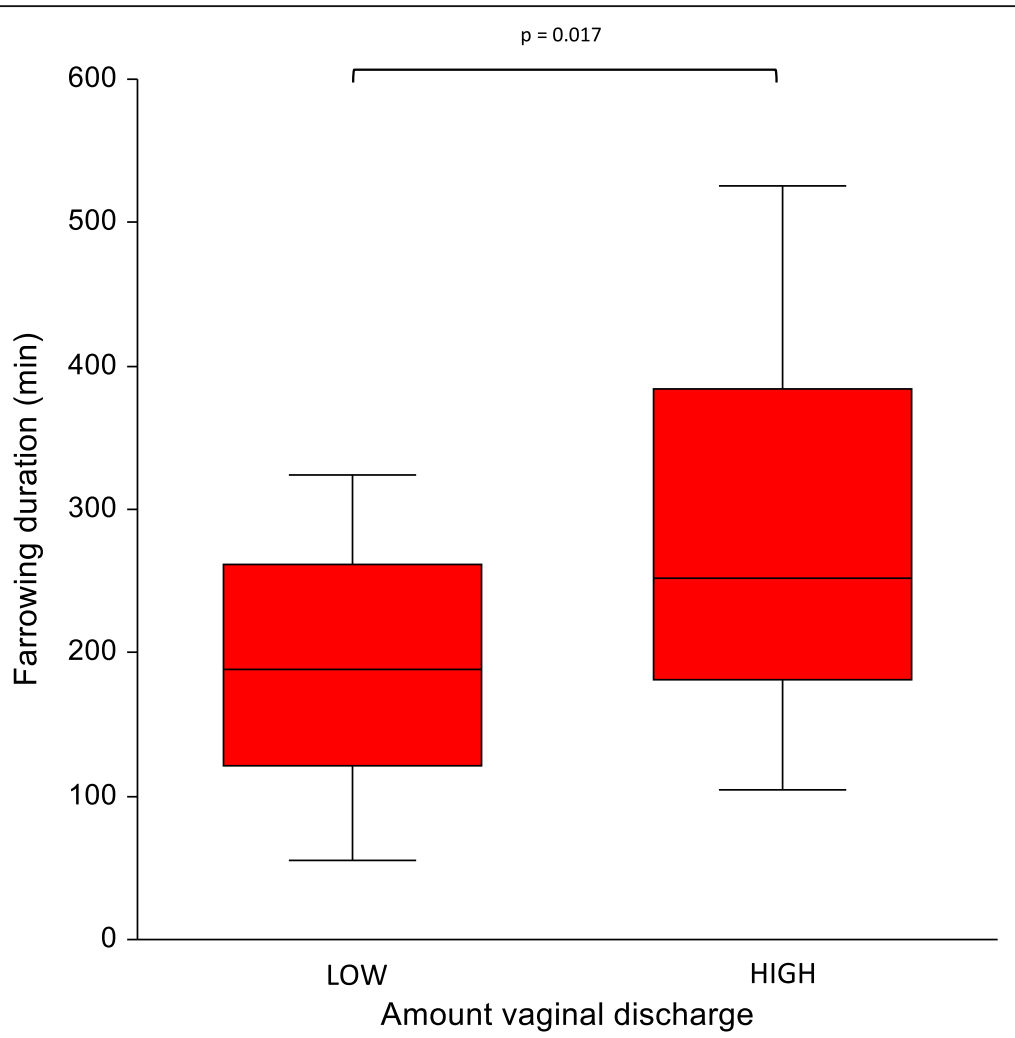

Fig. 9 Comparison of sows that had a low amount of vaginal discharge (LOW) and sows that hat a high amount of vaginal discharge (HIGH) evaluated daily in the first five days post-partum with the farrowing duration

presented in Fig. 10. Finally, no significant difference in the body temperature of sows, which received obstetrics during parturition, compared to sows without birth assistance were detected over the sampling period.

The vaginal $\mathrm{pH}$-value of 46 sows on first- and fifth-day post-partum and of 47 sows on the other sampling days were measured with two different test systems (strip and swab test). The data of each test system over the sampling period are presented in Figs. 11 and 12. In $74.9 \%$ of the samples the same $\mathrm{pH}$-value was obtained in the same individuum using different tests. Hence, no significant difference between the two test systems could be determined. A significant difference in the $\mathrm{pH}$-values between the second (strip: $6.6 \pm 0.4$; swab: $6.7 \pm 0.3$ ) and fourth day (strip: $6.9 \pm 0.4$; swab: $6.9 \pm 0.2$; strip: $p=$ 0.006 and swab: $p=0.0003$ ) and the second and fifth day post partum (strip: $6.8 \pm 0.3$; swab: $6.8 \pm 0.2$; strip: $\mathrm{p}=$ 0.025 and swab: $\mathrm{p}=0.013$ ) was detected.

A significant difference among the $\mathrm{pH}$-value of the vagina tested with the strip and the swab was detected between sows with no signs of vaginal discharge $(\mathrm{p}=$ $0.0118)$ and slight vaginal discharge $(\mathrm{p}=0.0109)$. Sows with no vaginal discharge had a lower vaginal $\mathrm{pH}$-value $(6.7 \pm 0.4 ; 6.8 \pm 0.2)$ compared to sows with a slight

Table 1 Farrowing duration clustered by parity in relation to the amount (LOW = sum of the scoring over five days $\leq 2 ; \mathrm{HIGH}=$ sum of the scoring over five days $\geq 3$ ) over 5 days post-partum

\begin{tabular}{|c|c|c|c|c|c|c|c|c|c|}
\hline & \multirow{2}{*}{\multicolumn{3}{|c|}{ All }} & \multicolumn{6}{|c|}{ Vaginal discharge } \\
\hline & & & & \multicolumn{3}{|c|}{ LOW } & \multicolumn{3}{|c|}{ HIGH } \\
\hline & $\begin{array}{l}\text { Median } \\
\text { (minutes) }\end{array}$ & $\begin{array}{c}\text { Min-Max } \\
\text { (minutes) }\end{array}$ & $N$ & $\begin{array}{l}\text { Median } \\
\text { (minutes) }\end{array}$ & $\begin{array}{c}\text { Min-Max } \\
\text { (minutes) }\end{array}$ & $\mathbf{N}$ & $\begin{array}{c}\text { Median } \\
\text { (minutes) }\end{array}$ & $\begin{array}{c}\text { Min-Max } \\
\text { (minutes) }\end{array}$ & $\mathbf{N}$ \\
\hline All sows (parity 1-11) & 211 & $55-525$ & 48 & 188.5 & $55-324$ & 28 & 252.5 & $104-525$ & 20 \\
\hline Parity Group A & 161 & $104-512$ & 13 & 171.5 & $105-277$ & 8 & 161 & $104-512$ & 5 \\
\hline Parity Group B & 190 & $55-525$ & 15 & 185 & $55-302$ & 10 & 365 & $178-525$ & 5 \\
\hline Parity Group C & 224 & $96-324$ & 8 & 211 & $96-324$ & 6 & 237.5 & $235-240$ & 2 \\
\hline Parity Group D & 255 & $110-427$ & 12 & 205 & $110-274$ & 4 & 275 & $151-427$ & 8 \\
\hline
\end{tabular}


Table 2 Amount of vaginal discharge, body temperature and feed intake in relation to the days post-partum

\begin{tabular}{|c|c|c|c|c|c|}
\hline \multirow[t]{2}{*}{ Days post-partum } & \multirow[t]{2}{*}{ Vaginal Discharge } & \multirow[t]{2}{*}{$\mathrm{N}$} & \multicolumn{2}{|l|}{ Temperature } & \multirow{2}{*}{$\begin{array}{l}\text { Feed intake } \\
\%(n / N)\end{array}$} \\
\hline & & & Median $\left({ }^{\circ} \mathrm{C}\right)$ & $\operatorname{Min}-\operatorname{Max}\left({ }^{\circ} \mathrm{C}\right)$ & \\
\hline \multirow[t]{4}{*}{1} & Over all & 46 & 38.8 & $37-40.7$ & $89.1(41 / 46)$ \\
\hline & 0 & 42 & 38.8 & $37-40.7$ & $90.5(38 / 42)$ \\
\hline & 1 & 1 & 37.7 & & $100.0(1 / 1)$ \\
\hline & 2 & 3 & 38.9 & $38.5-38.9$ & $66.6(2 / 3)$ \\
\hline \multirow[t]{4}{*}{2} & Over all & 47 & 38.5 & $36.5-41.1$ & $93.6(44 / 47)$ \\
\hline & 0 & 25 & 38.5 & $37-41.1$ & $92.0(23 / 25)$ \\
\hline & 1 & 11 & 38.5 & $36.5-39.9$ & $90.9(10 / 11)$ \\
\hline & 2 & 11 & 38.8 & $37.8-40.9$ & $100.0(11 / 11)$ \\
\hline \multirow[t]{4}{*}{3} & Over all & 47 & 38.3 & $37.5-39.9$ & $97.9(46 / 47)$ \\
\hline & 0 & 26 & 38.4 & $37.5-39.9$ & $96.1(25 / 26)$ \\
\hline & 1 & 15 & 38.2 & $37.5-39.7$ & $100.0(15 / 15)$ \\
\hline & 2 & 6 & 38.4 & $38.1-38.8$ & $100.0(6 / 6)$ \\
\hline \multirow[t]{4}{*}{4} & Over all & 47 & 38.3 & $37-39.7$ & $95.7(45 / 47)$ \\
\hline & 0 & 24 & 38.3 & $37.6-39.7$ & $100.0(24 / 25)$ \\
\hline & 1 & 18 & 38.2 & $37-39.3$ & $94.4(17 / 18)$ \\
\hline & 2 & 5 & 39 & $37.8-39.3$ & $80.0(4 / 5)$ \\
\hline \multirow[t]{4}{*}{5} & Over all & 45 & 38.5 & $37.3-40.5$ & $97.8(45 / 46)$ \\
\hline & 0 & 35 & 38.5 & $37.3-40.5$ & $97.1(34 / 35)$ \\
\hline & 1 & 9 & 38.1 & $37.4-39.7$ & $100.0(10 / 10)$ \\
\hline & 2 & 1 & 40 & & $100.0(1 / 1)$ \\
\hline
\end{tabular}

vaginal discharge $(6.9 \pm 0.3 ; 6.9 \pm 0.2)$ In addition, a significant difference of the $\mathrm{pH}$-value tested with the strip between sows with a reddish vaginal discharge compared to sows with a yellowish $(\mathrm{p}=0.0212)$ and whitish vaginal discharge $(\mathrm{p}=0.0182)$ was detected. Sows with a reddish vaginal discharge had a lower vaginal $\mathrm{pH}$-value (6.6 \pm $0.6)$ compared to sows with yellowish $(6.9 \pm 0.4)$ and whitish $(6.9 \pm 0.3)$ vaginal discharge. This trend could also be observed in the $\mathrm{pH}$-values obtained with the vaginal swab, but no significant difference was confirmed.

\section{Analysis of vaginal discharge traits on subsequent reproductive performance}

In this study, 6 out of 43 sows (13.9\%) returned to oestrus within six weeks after the subsequent mating. No significant difference between the return to oestrus rate could be determined among the LOW (4 out of 25 sows; $16.0 \%$ ) and HIGH (2 out of 18 sows; $11.1 \%$ ) group. The total born piglets and live-born piglets at the subsequent farrowing in sows with LOW vaginal discharge $(15.0 \pm 3.8 ; 14.3 \pm 3.6)$ was increased compared to the group with $\mathrm{HIGH}$ amount of vaginal discharge $(14.3 \pm 3.1 ; 13.7 \pm 3.3)$, but no significance could be confirmed. Furthermore, no other significant difference between the other parameters of the vaginal discharge and the subsequent reproductive performance was detected.

\section{Discussion}

This is the first study that evaluates different point-ofcare tests in parallel to characterize the lochia of sows after parturition in a free farrowing system. Furthermore, the characteristics of the vaginal discharge were associated with the subsequent reproductive performance of the same sows. The study design included a stratified random allocation blocked by parity to control the parity effect. Only the principal investigator conducted the sampling of the vaginal discharge and the post-partum parameters to reduce the observer bias. Although 48 sows were included in this observational study, only 39 sows showed signs of vaginal discharge. It might be that some sows with vaginal discharge were overlooked, because the evaluation was made once a day only. However, to minimize false negative results in sows, the observation was conducted early in the morning during the resting period of the sows to avoid urination before sampling. Since the sample size, especially per parity, was relatively small, only descriptive analyses for the parity were conducted. However, further analyses with all animals were conducted to minimize the detection bias. Another limitation of this study is that only sows from one farm in Switzerland were examined. Nonetheless, the results of this study are novel and of major interest for managing and optimizing the post farrowing 


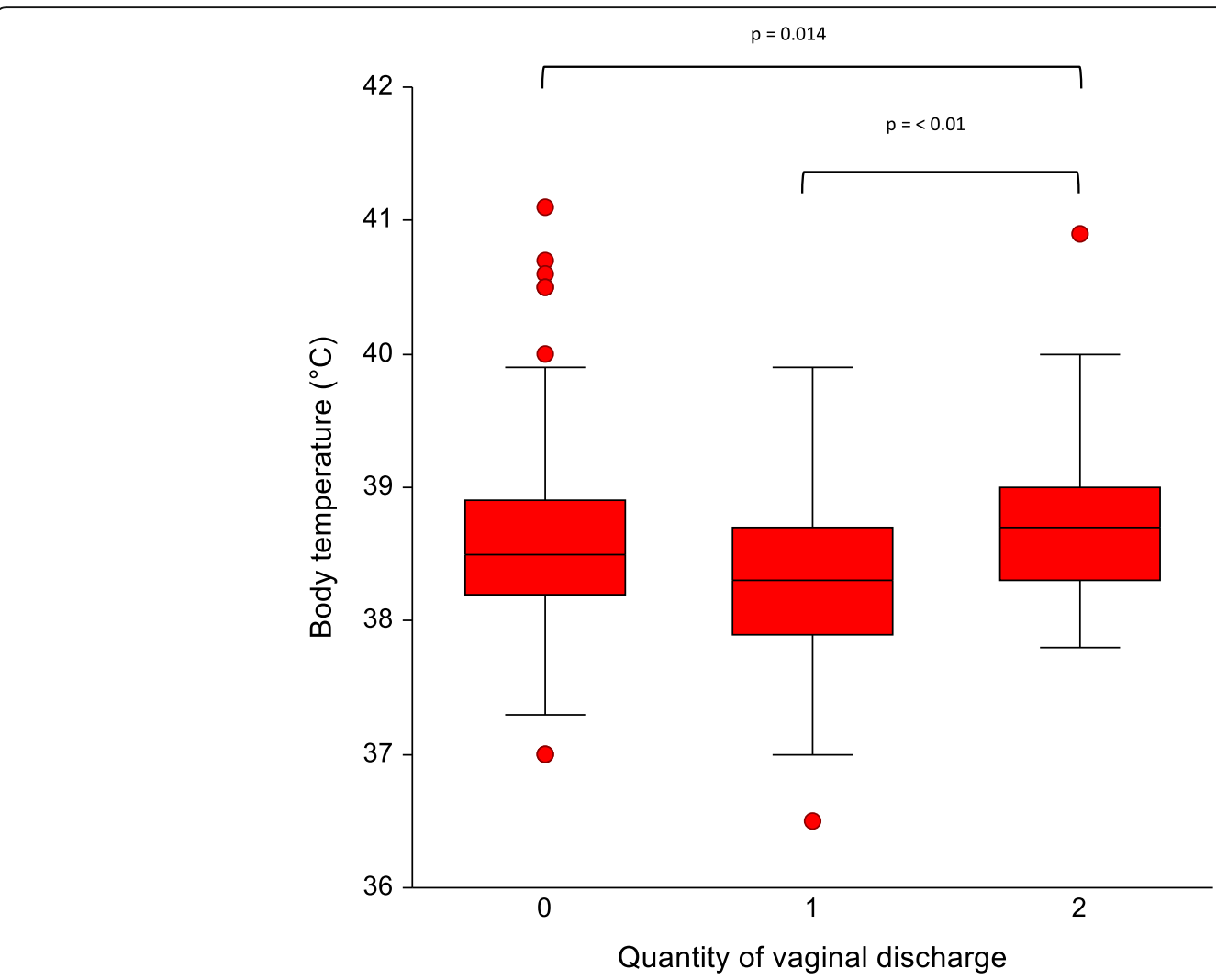

Fig. 10 Comparison of the quantity of vaginal discharge evaluated daily in the first five days post-partum with the body temperature of sows. The quantity of the vaginal discharge was categorized into score 0 (no signs of vaginal discharge), score 1 (slight vaginal discharge) and score 2 (severe vaginal discharge, tail and perineal region contaminated with vaginal discharge)

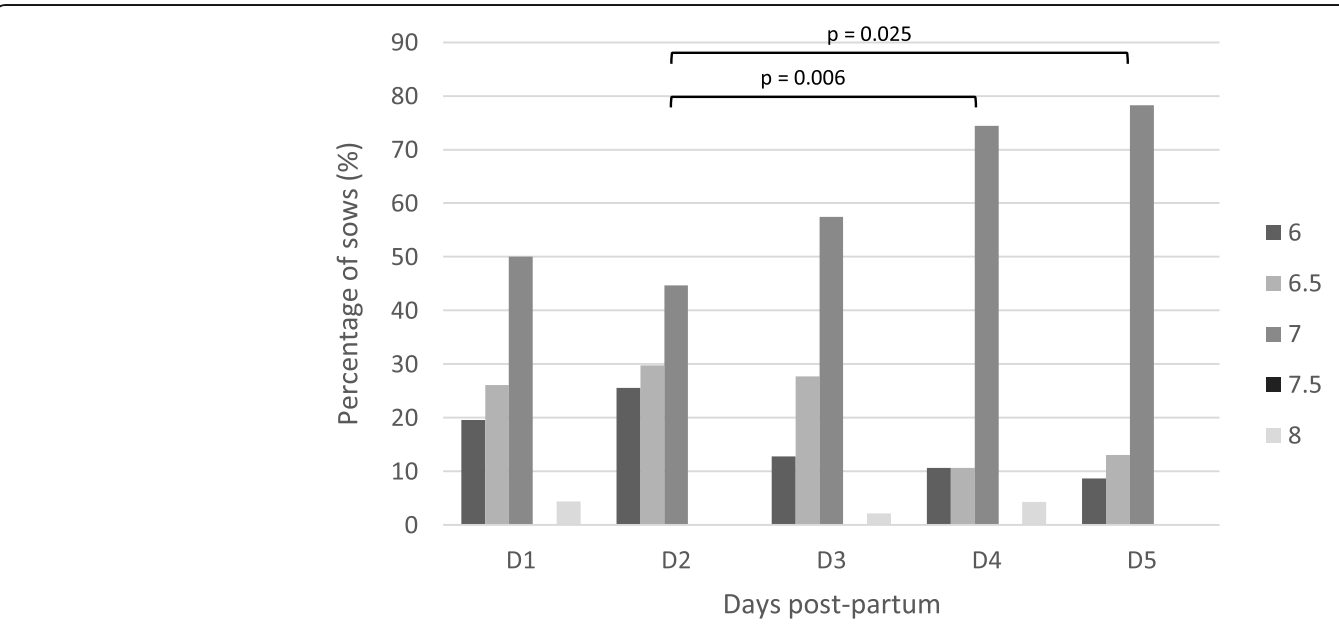

Fig. 11 Distribution of the percentage of sows according to $\mathrm{pH}$-value of the vagina tested with a strip system from day 1 to day 5 after parturition 


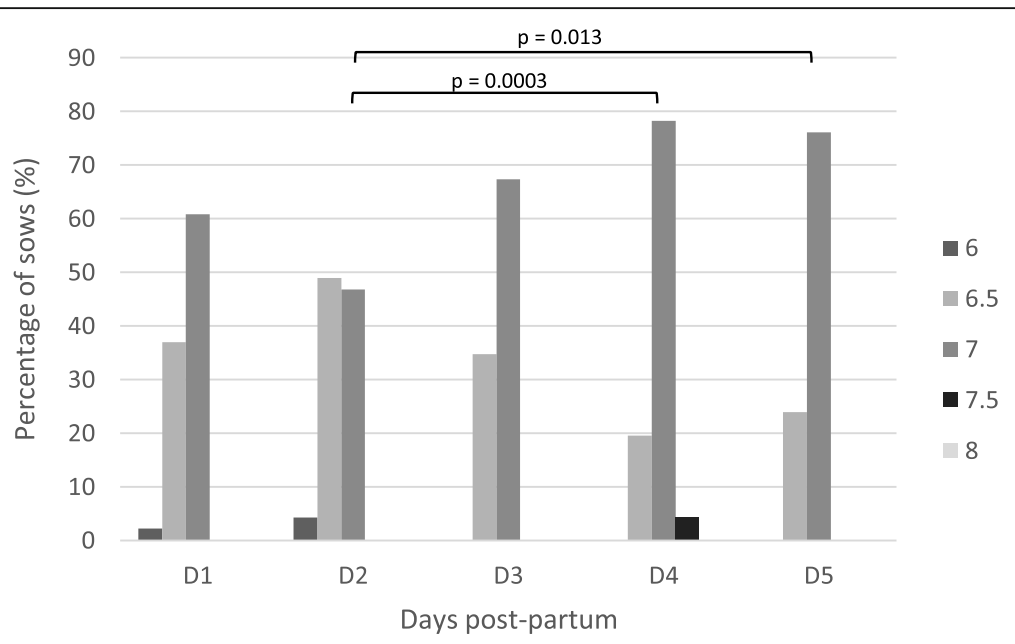

Fig. 12 Distribution of the percentage of sows according to $\mathrm{pH}$-value of the vagina tested with a swab system from day 1 to day 5 after parturition

phase in free farrowing sows. In addition, the sampling time points, and the evaluated test systems used in this study can certainly be implemented in sow herds with different housing systems and not only those with free farrowing.

In the present study, the prevalence of vaginal discharge throughout the different sampling days was lower compared to recent studies, although slight amount of vaginal discharge was included in the present data set too. Moreover, not only presence, but also colour of the vaginal discharge was evaluated. In this study, $8.3 \%$ of the sows on day $1,48.0 \%$ on day 2 and $43.8 \%$ on day 3 postpartum showed vaginal discharge, which is slightly differing from literature that reports a prevalence of 70.3 and $55.6 \%$ on day $1,53.1$ and $69.1 \%$ day 2 and $50.6 \%$ on day 3 , respectively $[11,12]$. The authors hypothesize that the reason for these differences in the prevalence of vaginal discharge is due to the free farrowing housing system, although the different assessments of the prevalence of vaginal discharge might limit the comparison of the results. However, in free farrowing system, sows can better express their natural behaviour, which presumably prevents from dysfunction of the endocrine regulation and decreases the stress level during parturition compared to systems with crated sows. Furthermore, sows in a free farrowing system have a higher average oxytocin concentration during the post-expulsion compared to crated sows [20] and thereby uterine clearance directly after farrowing is more likely. In order to scientifically proof this hypothesis, further investigations are needed.

In contrast to two studies $[8,21]$, where post parturient vaginal discharge was associated with parity, no significant difference between the different parities was detected in this study. However, the sample size per parity group is limited, and the housing system might have influenced the results, especially on day 1 , where the effect of free movement might have the greatest impact.

In accordance with previous reports [11, 12, 22], the amount of vaginal discharge positively correlated with the body temperature in sows. Physiologically, the body temperature increases and remains high during and after farrowing [23] due to nest-building activity, development of mammary glands and uterine contractions. In addition, the farrowing procedure initiates an inflammatory process evidenced by a rise of interleukins [3]. However, bacterial infections after parturition in the urogenital tract also lead to an increase of IL- 6 and tumour necrosis factor alpha in the blood, causing a rise in body temperature [3]. Therefore, it can be concluded that also in free farrowing sows the amount of vaginal discharge is a feasible parameter to evaluate uterine health after farrowing.

Data of the vaginal $\mathrm{pH}$-value of sows after parturition are lacking in current literature. This study observed that the $\mathrm{pH}$-value of the vagina is altering during the first days post-partum. A significant difference of the vaginal $\mathrm{pH}$-value between day $2(\mathrm{pH}=6.6)$ and day 4 and day 5 , respectively, $(\mathrm{pH}=6.9 ; \mathrm{pH}=6.8)$ after farrowing was observed. A study in miniature pigs showed that the vaginal $\mathrm{pH}$-value is neutral $(\mathrm{pH}=\sim 7)$ in sexually mature animals [24], like in our study on the last two sampling days. However, on the first days a more acidic $\mathrm{pH}$-value was seen, which is in line with results from human medicine, revealing an acidic vaginal secretion directly after labour [21]. Sows with a reddish vaginal discharge had a more acidic vaginal discharge compared to sows with whitish and yellowish vaginal secretion. It is hypothesised that sows with whitish and yellowish vaginal discharge have a higher risk for uterine disorders, due to changes of the microbial flora and inflammatory reactions [25]. This is in line with results of the 
cytological investigation, especially demonstrated by the amounts of neutrophil granulocytes. It is known from literature that sows with a higher number of polymorphonuclear cells are more prone to develop a puerperal endometritis [26]. However, further investigations are needed to prove these findings on a larger scale. In case of confirmation, these parameters could be reliable for diagnostic purposes and, thereby, help to detect postpartal disorders and enable improvement in sow and piglet health.

Hence, no correlation between parameters of the vaginal discharge and farrowing traits with the cell count of vaginal discharge could be detected, the PortaSCC ${ }^{\circ}$ test, established for milk samples in cows, might not be an adequate test kit for vaginal discharge.

Similarly to other studies, where sows were housed in crates $[6,10,13]$ a longer farrowing duration (Median: 252 vs. Median: 188) leads to a higher amount of vaginal discharge in free farrowing sows. Interestingly, the median farrowing duration of sows with a higher amount of vaginal discharge in the early puerperium is beyond the established cut-off value of $300 \mathrm{~min}$ [5] in crated sows. Hence, it can be discussed, if the cut-off value of farrowing duration needs to be adjusted according to the housing conditions of sows. In order to scientifically evaluate the optimal farrowing duration in free farrowing sows, further investigations are warranted.

Like in previous studies, obstetrical intervention positively correlated with the amount of vaginal discharge over the five sampling days. However, no significant differences in the body temperature and feed-intake between treated and non-treated sows were confirmed. This is in contrast with current literature. A potential explanation for these discrepancies is that the housing system influences the uterine health especially that of free farrowing sows. The physical movement after parturition might positively influence uterine involution, which is also described in the human medicine $[27,28]$. Hence, the free movement supports the physiological uterine clearance of the sow by hasting the uterine involution. Uterine contractility increases and leads to an elimination of intrauterine fluid, which reduces the risk of bacterial infection and consequently minimizes the risk of fever, reduced feed intake and other post-partum disorders. This assumption is in line with a recent study showing no influence of obstetrical intervention on the uterine involution evaluated with ultrasonography in free farrowing sows [29].

In the present study only a trend between the next litter size and the amount of vaginal discharge was detected, revealing that sows with a high amount of vaginal discharge have less total and/or stillborn piglets. This is in line with an older study, where only few significant differences between the reproductive performance and the duration of vaginal discharge was described [25]. However, it can be speculated that an early and adequate treatment of sow with high amount of vaginal discharge can support the uterine clearance and therefore increases the reproductive performance in the next litter.

\section{Conclusions}

The present study provides novel findings and original results to characterise the post parturient vaginal discharge by means of several point of care tests in free farrowing sows. In comparison with the recent literature of crated sows, the prevalence of post parturient vaginal discharge in free farrowing sows is reduced, which consequently minimizes the risk for fever, reduced feed intake and other post-partum disorders. However, a prolonged farrowing duration and obstetrical intervention significantly increases the amount of vaginal discharge. Nevertheless, it seems that the amount of vaginal discharge alone is not a valuable predictor for the performance of sows during their next gestation but might be an indicator for an acute endometritis. This indicator in combination with the colour of the vaginal discharge and the vaginal $\mathrm{pH}$-value might help to detect postpartal disorders early after farrowing and therefore enable improvement of sows' and piglets' health. In summary, the different parameters of the vaginal discharge determined by means of point-of-care tests might be useful to strengthen a presumptive diagnose of endometritis in sows during the first five days after parturition.

\section{Acknowledgements \\ The authors thank the farmer and his family for their collaboration.}

\section{Authors' contributions}

Conceptualization, Alexander Grahofer and Heiko Nathues; Data curation, Alexander Grahofer and Tara Mäder; Formal analysis, Alexander Grahofer; Funding acquisition, Heiko Nathues; Investigation, Alexander Grahofer and Tara Mäder; Methodology, Alexander Grahofer; Project administration, Alexander Grahofer; Resources, Heiko Nathues; Supervision, Heiko Nathues; Visualization, Alexander Grahofer; Writing - original draft, Alexander Grahofer; Writing - review \& editing, Tara Mäder and Heiko Nathues. The authors read and approved the final manuscript.

Funding

This research received no external funding.

Availability of data and materials

All the data are presented in the main paper and accompanying figures.

\section{Declarations}

Ethics approval and consent to participate

This study was conducted according to the Swiss law for Animal Welfare and approved by the the cantonal veterinary office of Solothurn (Licence Nr. SO 18/01 29818). In addition an informed consent was signed by the farmer.

Consent for publication

Not applicable.

Competing interests

There are no competing interests of any of the authors that could inappropriately influence or bias the content of the paper. 
Received: 17 February 2021 Accepted: 29 April 2021

Published online: 20 May 2021

\section{References}

1. Koketsu Y, Tani S, lida R. Factors for improving reproductive performance of sows and herd productivity in commercial breeding herds. Porc Heal Manag [Internet]. Porcine Health Management; 2017;3:1-10. Available from: https://doi.org/10.1186/s40813-016-0049-7.

2. Peltoniemi O, Oliviero C, Yun J, Grahofer A, Björkman S. Management practices to optimize the parturition process in the hyperprolific sow. J Anim Sci. 2020;98:96-106.

3. Kaiser M, Jacobsen S, Andersen PH, Bækbo P, Cerón JJ, Dahl J, et al. Hormonal and metabolic indicators before and after farrowing in sows affected with postpartum dysgalactia syndrome. BMC Vet Res BMC Veterinary Research. 2018;14:1-15.

4. Oliviero C, Junnikkala S, Peltoniemi O. The challenge of large litters on the immune system of the sow and the piglets. Reprod Domest Anim [Internet]. Blackwell Publishing Ltd; 2019 [cited 2020 Feb 11];54:12-21. Available from: https://doi.org/10.1111/rda.13463.

5. Oliviero $C$, Kothe $S$, Heinonen $M$, Valros A, Peltoniemi O. Prolonged duration of farrowing is associated with subsequent decreased fertility in sows. Theriogenology [Internet]. 2013 [cited 2017 Jul 10];79:1095-9. Available from: http://www.ncbi.nlm.nih.gov/pubmed/23481671.

6. Björkman S, Oliviero C, Kauffold J, Soede NM, Peltoniemi OAT. Prolonged parturition and impaired placenta expulsion increase the risk of postpartum metritis and delay uterine involution in sows. Theriogenology. 2018;106:8792.

7. Nachreiner R, Ginther O. Gestational and periparturient periods of sows: effects of altered environment, withholding of bran feeding, and induced mastitis on serum chemical, hematologic, and clinical variables. Am J Vet Res. 1972;33:2221-31.

8. Hermansson I, Einarsson S, Larsson K, Bäckström L. On the agalactia post partum in the sow. A clinical study. Nord Vet Med. 1978;30:465-73.

9. Morkoç A, Bäckström L, Lund I, Smith A. Bacterial endotoxin in blood of dysgalactic sows in relation to microbial status of uterus, milk, and intestine. J Am Vet Med Assoc. 1983;183:786-9.

10. Nam NH. Risk factors for prolonged postparturient vaginal discharge in sows. Thai J Vet Med. 2020;50:57-63.

11. Tummaruk P, Sang-Gassanee K. Effect of farrowing duration, parity number and the type of anti-inflammatory drug on postparturient disorders in sows: a clinical study. Trop Anim Health Prod [Internet]. 2013 [cited 2016 Feb 11]; 45:1071-7. Available from: http://www.ncbi.nlm.nih.gov/pubmed/23143739.

12. Tummaruk $P$, Pearodwong P. Postparturient disorders and backfat loss in tropical sows associated with parity, farrowing duration and type of antibiotic. Trop Anim Health Prod. 2015;47:1457-64.

13. Hoy S. Investigations on the effects of puerperal diseases in sows on the fertility. Arch Anim Breed. 2003:46:341-6.

14. Muirhead M. Epidemiology and control of vaginal discharges in the sow after service. Vet Rec. 1986;119:233-5.

15. Meredith M. Non-specific bacterial infections of the genital tract in female pigs. Pig Vet J. 1991;27:110-21.

16. de Winter PJJ, Verdonck M, de Kruif A, Devriese LA, Haesebrouck F. Endometritis and vaginal discharge in the sow. Anim Reprod Sci. 1992;28: $51-8$.

17. Grahofer A, Björkman S, Peltoniemi O. Diagnosis of endometritis and cystitis in sows: use of biomarkers. J Anim Sci. 2020;98:107-16.

18. Bará MR, Cameron RDA. No Title. Proc Int Pig Vet Soc Congr. 1996. p. 574

19. Muirhead MR, Alexander TJL. Managing pig health and the treatment of disease: a reference for the farm. Sheffield S35 OBP: 5 M Enterprises Ltd., PO Box 233.; 1997.

20. Oliviero C, Heinonen M, Valros A, Hälli O, Peltoniemi OAT. Effect of the environment on the physiology of the sow during late pregnancy, farrowing and early lactation. Anim Reprod Sci [Internet]. 2008 [cited 2015 Sep 2];105:365-77. Available from: http://www.sciencedirect.com/science/a rticle/pii/S0378432007001200

21. Brabin L, Roberts SA, Fairbrother E, Mandal D, Higgins SP, Chandiok S, et al. Factors affecting vaginal $\mathrm{pH}$ levels among female adolescents attending genitourinary medicine clinics. Sex Transm Infect. 2005;81:483-7.

22. Tummaruk P. Post-parturient disorders and backfat loss in tropical sows in relation to backfat thickness before farrowing and postpartum intravenous supportive treatment. Asian-Australasian J Anim Sci. 2013;26:171-7.
23. King GJ, Willoughby RA, Hacker RR. Fluctuations in rectal temperature of swine at parturition. Can Vet J. 1972;13:72-4.

24. Lorenzen E, Follmann F, Jungersen G, Agerholm JS. A review of the human vs. porcine female genital tract and associated immune system in the perspective of using minipigs as a model of human genital Chlamydia infection. Vet Res [Internet]. Veterinary Research; 2015;46:1-13. Available from: https://doi.org/10.1186/s13567-015-0241-9.

25. Mania-Pramanik J, Kerkar SC, Mehta PB, Potdar S, Salvi VS. Use of vaginal pH in diagnosis of infections and its association with reproductive manifestations. J Clin Lab Anal. 2008;22:375-9.

26. Carabin H, Desnoyers M, Vaillancourt D, Martineau GP. Influence of vulvar hygiene on cytology of vaginal smears after sham artificial insemination in sows. Can J Vet Res. 1995:59:193-6.

27. Berensmann I, Klein S, Reese S, Erhard M, Patzkéwitsch D. A comparison of different farrowing systems - Part 1: Effects on the activity of the sow. Tierärztliche Prax Ausgabe G Großtiere /. Nutztiere. 2018;46:291-7.

28. Evenson KR, Mottola MF, Owe KM, Rousham EK, Brown WJ. Summary of international guidelines for physical activity after pregnancy. Obstet Gynecol Surv. 2014;69:407-14.

29. Meile A, Nathues H, Kauffold J, Grahofer A. Ultrasonographic examination of postpartum uterine involution in sows. Anim Reprod Sci. 2020;219.

\section{Publisher's Note}

Springer Nature remains neutral with regard to jurisdictional claims in published maps and institutional affiliations.
Ready to submit your research? Choose BMC and benefit from:

- fast, convenient online submission

- thorough peer review by experienced researchers in your field

- rapid publication on acceptance

- support for research data, including large and complex data types

- gold Open Access which fosters wider collaboration and increased citations

- maximum visibility for your research: over $100 \mathrm{M}$ website views per year

At BMC, research is always in progress.

Learn more biomedcentral.com/submissions 